\title{
Detection of the Sentinel Lymph Node With Hybrid Tracer (ICG-[99mTc]Tc-albumin Nanocolloid) in Intermediate and High Risk Endometrial Cancer. A Feasibility Study
}

Nuria Sánchez Izquierdo

Hospital Clinic de Barcelona https://orcid.org/0000-0002-8565-1552

Sergi Vidal-Sicart

Hospital Clinic de Barcelona

\section{Francisco Campos}

Hospital Clinic de Barcelona

Aureli Torné

Hospital Clinic de Barcelona

\section{Martina Aida Angeles}

Institut Claudius Regaud: Institut Universitaire du Cancer Toulouse Oncopole

Federico Migliorelli

Centre Hopital Intercommunal Des Vallees De L Ariege Pamiers

Meritxell Munmany

Hospital Clinic de Barcelona

\section{Adela Saco}

Hospital Clinic de Barcelona

Berta Diaz-Feijoo

Hospital Clinic de Barcelona

Ariel Glickman

Hospital Clinic de Barcelona

Jaume Ordi

Hospital Clinic de Barcelona

Andrés Perissinotti

Hospital Clinic de Barcelona

Marta del Pino

Hospital Clinic de Barcelona

Pilar Paredes ( $\triangle$ pparedes@clinic.cat)

Hospital Clinic de Barcelona 


\section{Research Article}

Keywords: Hybrid tracer (ICG-[99mTc]Tc-albumin nanocolloid), sentinel lymph node, endometrial cancer, lymphatic mapping, radiotracer, ICG

Posted Date: August 5th, 2021

DOl: https://doi.org/10.21203/rs.3.rs-773719/v1

License: (c) (i) This work is licensed under a Creative Commons Attribution 4.0 International License.

Read Full License 


\section{Abstract}

Purpose: Indocyanine green (ICG) is frequently used for the detection of the sentinel lymph node (SLN) in gynecology, but it carries the loss of the presurgical SLN mapping provided by [ ${ }^{99 \mathrm{~m}} \mathrm{Tc}$ ]-based colloids. Hybrid tracers such as ICG-[ $\left.{ }^{99 \mathrm{~m}} \mathrm{Tc}\right] \mathrm{Tc}$-albumin nanocolloid combine the benefits of both components. The aim of this study was to evaluate the feasibility and applicability of this hybrid tracer injected by transvaginal ultrasound-guided myometrial injection of radiotracer (TUMIR) approach in the detection of SLNs in patients with intermediate- and high-risk EC.

Methods: Fifty-two patients with intermediate- and high-risk EC underwent SLN biopsy after injection of a hybrid tracer using the TUMIR approach, followed by pelvic and paraaortic lymphadenectomy. SLNs were detected preoperatively by lymphoscintigraphic study and intraoperatively by gamma probe and nearinfrared (NIR) optical laparoscopic camera.

Results: Preoperative lymphatic drainage was obtained in $69 \%$ and intraoperative detection in $71.4 \%$ of patients. A total of 146 SLNs (4.17 SLNs/patient) were biopsied. Pelvic bilateral detection was observed in $57 \%$ of the women and paraaortic drainage in $34 \%$ of the patients. The radioactive component allowed the detection of SLN in $97.1 \%$ of the patients, while the fluorescent component detected $80 \%$. In more than $17 \%$ of the patients with intraoperative detection, SLNs were detected only by the radioactive signal. Lymph node metastasis were identified in $14.3 \%$ of patients submitted to SLNB. The sensitivity and negative predictive value for metastatic involvement were $100 \%$.

Conclusion: TUMIR injection of a hybrid tracer in patients with intermediate- and high- risk EC combines the benefits of the radiotracer and the fluorescence methods with a single tracer. The method increases the paraaortic detection rate and allows a potential increase in SLN detection. Notwithstanding, based on our findings, the radioactive component of the hybrid tracer cannot be obviated.

\section{Introduction}

Endometrial cancer (EC) is the most frequent gynecological malignancy in high-income countries. ECs are classified as low, intermediate and high risk based on their risk of lymph node and distant metastases. As in most solid tumors, lymph node infiltration is the most important prognostic factor, and therefore, systematic pelvic and paraaortic lymphadenectomy is indicated in high-risk tumors and is considered in those of intermediate risk [1]. However, the role of systematic lymphadenectomy has recently been questioned because it has a significant associated morbidity and results in an overtreatment of up to $80 \%$ of women [2]. Sentinel lymph node biopsy (SLNB) has been developed as an alternative to systematic lymphadenectomy, associated to a low morbidity rate. Furthermore, SLN ultrastaging, which involves serial sections and the use of immunohistochemical stains, allows increasing the sensitivity for metastasis. The guidelines of the National Comprehensive Cancer Network recommend SNLB in patients with low-risk EC [3]. Recent evidence indicates that SLNB achieves a detection rate of $89 \%$ and a false 
negative rate of $11.5 \%$ in patients with intermediate- and high-risk EC. [4] However, its indication in these women with intermediate- and high-risk EC is still under debate.

Traditionally, SLNB in patients with gynecological tumors has been performed using a technetium-based radiotracer ([99m Tc]Tc-albumin nanocolloid), either alone or combined with blue dye. In these cases the radiotracer allows obtaining preoperative lymph node mapping, which increases the detection of SLN [5]. Nonetheless, the SLN detection rate is lower in patients with EC than in other gynecological tumors. Reduced SLN detection in these patients has been associated to older age, tumor size bigger than $2 \mathrm{~cm}$ and low radiotracer volumes [6]. The combination with blue dye does not seem to significantly increase the rate of detection in these patients with $\mathrm{EC}[7,8]$.

To solve these problems, the use of fluorescent, non-radioactive tracers has been introduced. Among these tracers, indocyanine green (ICG), which can be detected intraoperatively, has been used as a single tracer in gynecological tumors of the endometrium $[9,10]$, cervix [11,12] and vulva [13]. However, unlike radiotracer, ICG is not retained by the macrophages of the lymph nodes and therefore, the injection must be performed intraoperatively due to its rapid drainage, precluding obtaining a preoperative lymph node map for planning surgery.

In the last decade hybrid tracers (ICG-[ $\left.{ }^{99 \mathrm{~m}} \mathrm{Tc}\right] \mathrm{Tc}$-albumin nanocolloid) have been developed. These hybrid tracers combine the advantages of the two modalities [14-16]: the radiotracer allows presurgical lymphatic mapping and provide an acoustic signal through the gamma detector probe, whereas ICG allows the visualization of the SLN during surgery. This hybrid tracer has shown to be more efficient and accurate to localize the SLN compared with blue dye or the two components (ICG and radiotracer) used alone [14-16] in urological neoplasms such as cancers of the prostate gland [17] and penis [14]. The hybrid tracer has also been successfully used in gynecological neoplasms, such as vulvar [18] and cervical cancer [19], with a high rate of SLN detection (96-100\%), but has not been evaluated in EC.

The aim of this study was to evaluate the feasibility of using a single hybrid tracer and its performance to detect SLN in patients with high and intermediate risk EC.

\section{Materials And Methods}

\section{Patients and study design}

Patients with preoperative diagnosis of intermediate and high-risk EC submitted to SLNB and clinically indication in our center of complete pelvic and paraaortic lymphadenectomy between 2014 and 2019 were included in this study. The patients fulfilled at least one of the following inclusion criteria: 1) unfavorable histology (serous, clear cell or grade 3 endometrioid adenocarcinoma); 2) myometrial invasion $\geq 50 \%$ suspected by imaging techniques (magnetic resonance imaging (MRI) or 3D ultrasound); 3 ) involvement of the cervical stroma confirmed by biopsy or suspected by imaging techniques. 
Patients with the following criteria were excluded from our study: 1) contraindication for surgical staging; 2) metastatic disease suspected in the preoperative evaluation by computed tomography (CT) or MRI or confirmed histologically; 3 ) previous surgery or radiotherapy in the pelvic or paraaortic regions.

This retrospective study from prospectively collected data was approved by our Institutional Review Board (HCB/2019/0574) and written informed consent was obtained from all the patients included in the study prior to the administration of the hybrid tracer.

\section{Injection of the hybrid tracer}

The hybrid tracer was prepared in the Radiopharmacy Unit according to the usual methodology of our center [19]. Between 18 and 24 hours before surgery the patients were injected with a dose of $222 \mathrm{MBq}$ (6 $\mathrm{mCi}$ ) at a total volume of $4 \mathrm{ml}$ of hybrid tracer. Two $\mathrm{ml}$ of the hybrid tracer plus $1 \mathrm{ml}$ of physiological saline were injected into the anterior and posterior part of the myometrium to increase interstitial pressure in the tissue, separated by $1 \mathrm{ml}$ of air between the two injections to avoid in vivo dilution of the hybrid tracer.

This injection technique used was the transvaginal ultrasound-guided myometrial injection of radiotracer (TUMIR) approach, as previously described [6,20]. An Aspen (Siemens-Acuson Inc., MountainView, CA, USA) or Voluson ultrasound image equipment (Voluson v730Expert, General Electric, Germany), equipped with a vaginal probe coupled with a caliber 20 biopsy needle guide (Gallini Medical Devices, Mantova, Italy) was used for injection of the hybrid tracer. All the procedures were performed by an expert gynecologist together with a specialist in nuclear medicine (Figure 1).

\section{Preoperative lymphoscintigraphy}

Following the injection of the hybrid tracer, planar abdominal-pelvic images (matrix of $256 \times 256$, anterior and lateral views of $300 \mathrm{~s} /$ frame) were obtained using a single-head gamma camera (E-Cam, Siemens, Erlangen, Germany) or dual-head gamma camera (Infinia ${ }^{T M}$ Hawkeye ${ }^{T M} 4$; GE Healthcare Milwaukee, WI, USA), equipped with a low energy high resolution collimator. The images were obtained at 30 minutes and at 2-4 hours after the injection of the hybrid tracer.

Afterwards, single photon emission computed tomography (SPECT) images with CT for correction of attenuation and posterior fusion of the reconstructed image were acquired with two different dual-head gamma camera (Infinia ${ }^{\mathrm{TM}}$ Hawkeye ${ }^{\mathrm{TM}} 4$; GE Healthcare Milwaukee, WI, USA" (120 projections in a noncircular $360^{\circ}$ orbit, $15 \mathrm{~s} /$ projection in the matrix of $128 \times 128$ with a pixel size of $3.16 \times 3.16 \mathrm{~mm}^{2}$ ) using a CT image (matrix size 512 × 512, $140 \mathrm{kV}$ and $2.5 \mathrm{mAs}$ ) and a Symbia Intevo Bold (Siemens Healthineers, Erlangen, Germany) gamma camera was used (120 projections in a non-circular $360^{\circ}$ orbit, 30 s/projection, in a matrix of $128 \times 128$ with a pixel size of $3.30 \times 3.30 \mathrm{~mm}^{2}$ ), with CT image (matrix size $512 \times 512,130 \mathrm{kV}$ and 4D CARE Dose). 
In addition, volumetric reconstructions were performed using the Osirix Dicom viewer (Pixmeo SARL, Geneva, Switzerland) in an operating system based on Unix (MAC OS X, MacPro; Apple, Cupertino, CA) for obtaining a tridimensional presentation and improving anatomical localization of the SLNs (Figure 2).

The images were examined by two specialists in Nuclear Medicine and were discussed with the surgical team prior to surgery.

\section{Intraoperative SLN detection}

In all the patients, the detection of SLNs was made by laparoscopic surgery. The laparoscopic surgery was started by an intraperitoneal approach to rule out carcinomatosis. Then, a left retroperitoneal access was created to first localize the paraaortic SLNs.

For intraoperative localization of the SLN a laparoscopic gamma ray detector probe was used (Navigator; USSC, Norwalk, CT, USA), which is compatible with a laparoscopic trocar of $12 \mathrm{~mm}$ in diameter. For the detection of fluorescence, a specialized laparoscopic optical camera was used, which included a nearinfrared (NIR) image option (Karl Storz Image1 S ${ }^{\text {TM}}$; Karl Storz, Tuttlingen, Germany). This system allows obtaining images with white light and fluorescence in the NIR spectrum according to in vivo needs.

After SLNB, the number of counts emitted by the radiotracer and the intensity of the luminous emission of NIR ex vivo were collected using the same devices described for the in vivo detection (Figure 3).

A lymph node was considered a SLN when: a) it was the first node visualized in the lymphoscintigraphy or showed greater activity in the late planar images or was visualized in the SPECT/CT in a region other than in the planar lymphoscintigraphy; $b$ ) it was the most active node during surgery according to the gamma probe or previous lymphoscintigraphy; or c) it was fluorescent. Each side of the pelvis and the paraaortic area were considered independent lymphatic territories according to the Memorial Sloan Kettering Cancer Center algorithm[21]. The exact location of each SLN in relation to the pelvic, vena cava or aorta vessels was recorded. The number of SLN excised, their localization and their intraoperative detection signal were reported.

\section{Paraaortic lymphadenectomy, pelvic SLNB and hysterectomy}

After selective excision of paraaortic SLNs, systematic paraaortic lymphadenectomy was carried out. The procedure included removal of the lymph nodes located at the presacral, aortic bifurcation, precaval, preaortic and paraaortic areas, both below and above the inferior mesenteric artery up to the level of the left renal vein as the upper limit of dissection.

Lymphadenectomy continued through a transperitoneal approach to perform a selective excision of pelvic SLNs. The pelvic regions were carefully scanned with the gamma probe, angled laterally to avoid detection of radioactivity at the injection site. Then, a bilateral transperitoneal pelvic lymphadenectomy was performed, including the removal of external and internal iliac, obturator fossa, and common iliac 
lymph nodes. Finally, a total vaginal hysterectomy and bilateral salpingo-oophorectomy assisted by laparoscopy was performed.

\section{Histological evaluation of the lymph nodes}

The SLNs were cut into $2 \mathrm{~mm}$-thick serial sections, submitted completely for histology, and routinely embedded in paraffin. A first four micrometer-thick section was stained with hematoxylin and eosin (H\&E) and examined under a light microscope. If this first section was negative, four additional pairs of sections were performed at an interval of $200 \mu \mathrm{m}$ (ultrastaging). Of each pair, one section was stained with H\&E and the other using immunohistochemistry for cytokeratin 7 (Dako Pathology, Agilent, Santa Clara, CA, USA). Immunohistochemical studies were performed with the automated immunohistochemical system Autostainer Link $48^{\circledR}$, using the EnVision system (Dako).

The lymphadenectomy specimens were fixed in neutral-buffered formalin and macroscopically dissected to isolate all lymph nodes, which were cut into $2 \mathrm{~mm}$-thick sections following their largest diameter and routinely processed. Four micrometer-thick histological sections were obtained with a microtome, which were stained with $\mathrm{H} \& \mathrm{E}$ and examined under a light microscope.

Metastatic involvement was defined as at least one lymph node (either SLN or one non-SLN) positive for metastases detected either in the evaluation of the H\&E and/or the immunohistochemical section (in case of SLN). When present, the size of the metastasis was recorded. Isolated tumor cells and metastatic involvement less than $2 \mathrm{~mm}$ (micrometastases) were considered as low-volume metastases.

\section{Statistical analysis}

Categorical variables are described as absolute numbers and percentage, while continuous features are shown as mean and standard deviation (SD).

The number of SLNs retrieved during surgery was compared with the number of SLN identified in the planar lymphoscintigraphy and SPECT/CT. We also compared the number of SLN detected by each of the tracers, individually and simultaneously (ICG/Radiotracer/ICG+Radiotracer).

Categorical variables were evaluated by the Fisher exact test and continuous variables by the Student's ttest. The paired Student's t-test was used to evaluate the preoperative and postoperative detection rates.

The accuracy analysis of the hybrid detection of the SLN was carried out including exclusively the women with successful SLN resection (meaning that at least one SLN was intraoperatively identified and excised). The histological result of the SLN was contrasted with the result of the histopathological analysis of the nodes retrieved during lymphadenectomy. With these data, sensitivity, specificity and the positive predictive value (PPV) and negative predictive value (NPV) of the histological results of the SLN were calculated, as well as their respective 95\% confidence intervals (Cl), using the Wilson's method. Differences were considered significant at a $5 \%$ bilateral level, and all analyses were performed using Stata 13.1 (StataCorp, Texas). 


\section{Results}

A total of 52 patients were included in the analysis with preoperative intermediate ( 33 women) or highrisk (19 women) EC, assessed according to ESMO-ESGO-ESTRO Consensus Conference on EC Classification. The mean age of the patients was 63.6 years (SD 10.3). The histological characteristics and International Federation of Gynecology and Obstetrics stage of the patients included in the study are summarized in Table 1. There were no adverse reactions either during or after injection of the hybrid tracer.

\section{Preoperative detection: Lymphoscintigraphy (planar and SPECT/CT images)}

Drainage towards the pelvic and/or paraaortic lymph node chains was observed in $48.1 \%(25 / 52)$ of the patients in the planar lymphoscintigraphy images and in 69.2\% (36/52) in the tomographic images of SPECT/CT. Among the women in whom SLN were detected, bilateral pelvic SLN were visualized in $55.6 \%$ (20/36). In none of the women the drainage was exclusively paraaortic. Table 2 shows the distribution of the drainage.

In $30.8 \%(16 / 52)$ of the patients, drainage was not observed in the presurgical images. In three of these 16 women (6\%) peritoneal diffusion was observed, in other three $(6 \%)$ there was an important uptake in the bone marrow, and the remaining 10 patients (19\%) showed an absence of lymphatic drainage of the hybrid tracer.

Among the 36 patients showing drainage, 79 SLNs were observed in the planar images, with a mean of

2.19 (SD 1.92) per patient, and 138 SLNs were visualized in the SPECT/CT images with a mean of 3.83 (SD 2.75) per patient $(p<0.05)$.

\section{Intraoperative detection: Surgery}

During surgery, three patients (belonging to the group of patients with drainage) showed peritoneal carcinomatosis, so SLNB was ruled out. That results in 49 patients in whom SLNB was attempted. Among the 16 women without preoperative drainage and who underwent surgery, SLNs were intraoperatively detected in two patients. In the remaining 14 patients, the absence of lymphatic drainage was confirmed (26.9\%). At least one SLN was intraoperatively detected in 35 patients of the 49 , which means an intraoperative detection rate of $71.4 \%$ (35/49). These 35 women will comprise the group of patients for the accuracy analysis. These findings are shown in the flow chart of Figure 4.

A total of 146 SLNs were excised with a mean of 4.17 (SD 2.81) SLN per patient (146/35), with bilaterality of $57.1 \%(20 / 35)$. Paraaortic SLNs were biopsied in $34.3 \%(12 / 35)$ of the patients. No isolated paraaortic SLNs were biopsied. Figure 5a shows the topographic distribution of the SLNs.

In the analysis of SLN detection by tracer, of the 146 SLNs excised, $96.6 \%(141 / 146)$ showed radioactivity signal, and $71.9 \%$ (105/146) showed fluorescence signal. Among the 146 SLNs, $68.5 \%(100 / 146)$ of the 
SLNs biopsied, were detected by both components. In $28.1 \%$ (41/146), the SLNs were detected by the radioactive signal but we were not able to intraoperatively detect any emission of fluorescence of ICG, and in 3.4\% (5/146) of the SLNs, fluorescence but not gamma emission was detected. There was a significant difference when comparing the detection tool used (gamma probe vs NIR optical laparoscopic camera) $(p<0.001)$. The topographic distribution based on the signal detected is shown in Figure $5 b$.

In the analysis of SLN detection by patient, the radioactive component allowed the detection of SLNs in $97.1 \%(34 / 35)$ of the patients while the fluorescent component detected $80 \%(28 / 35)$. Among the 35 patients, in $20 \%(7 / 35)$ it was only one of the components and not both that allowed SLN detection. In $17.2 \%(6 / 35)$ of these patients, SLNs were detected only by the radioactive signal and in the remaining $2.8 \%(1 / 35)$ only one SLN with fluorescence emission was detected.

On comparing the SLNs pre-surgically visualized with those which were biopsied during surgery, there were no differences between the detection with SPECT/CT and surgical detection ( $p 0.83$ ), but there was a difference between planar and surgical detection and between planar and tomographic detection $(p<$ 0.05).

In six women, more SLNs were biopsied during surgery than those visualized in the preoperative images, representing an increase in the number of lymphatic territories studied. Three patients with unilateral preoperative drainage presented bilateral SLNs; in one of these patients one paraaortic SLN was also biopsied. In two patients without drainage in the SPECT/CT, at least one unilateral SLN was biopsied, and in one of these patients paraaortic SLNs were also biopsied. In the remaining patient with unilateral pelvic drainage in the SPECT/CT, paraaortic SLNs underwent biopsy also.

\section{Histological lymph node results}

Among the 35 patients in whom at least one SLN was biopsied, the histopathological study showed lymph node metastasis in $14.3 \%(5 / 35)$.

In the other 30 patients with negative SLNs, no metastatic node was found in the lymphadenectomy sample. SLN detection by the TUMIR approach for detecting nodal involvement in patients in whom intraoperative SLNB was achieved were $100 \%(95 \% \mathrm{Cl} ; 56.6-100 \%)$ for the sensitivity and PPV and $100 \%$ (95\% Cl; 88.6-100\%) for the specificity and NPV.

In three of the 14 patients without preoperative drainage and in whom intraoperative detection was not achieved, metastatic lymph nodes were observed in the lymphadenectomy; in two patients the lymph nodes were of pelvic localization while in the other patient they were only paraaortic.

\section{Discussion}

To date the value of hybrid tracer has not been evaluated in EC. In our series, this hybrid tracer was injected into the myometrium by the TUMIR approach for SLNB in patients with preoperative 
characteristics high- and intermediate-risk with the aim of improving lymphatic staging in this group of tumors.

The overall preoperative detection rate $(69 \%)$ was less than that reported with radiotracer injected by the TUMIR approach $(71 \%-82 \%)[6,20]$, as was the paraaortic preoperative detection rate of $36 \%$ compared to $41 \%-45 \%$ described in previous studies, which were not, in any case, exclusively paraaortic. However, we found a higher rate of preoperative bilateral pelvic detection (56\%) compared to the previously mentioned studies $(29 \%-37 \%)$.

The overall intraoperative detection rate was $71 \%$, similar to that reported with radiotracer only injected by the TUMIR approach (74\%)[20]. The intraoperative detection rate in the bilateral pelvic region was $57 \%$, being superior than in previous TUMIR studies (29\%), and in the paraaortic region SLNs were biopsied in $34 \%$ similar to other studies published describing the TUMIR approach [20]. This increase in the detection rate of intraoperative bilateral pelvic SLN detection could be explained by the hybrid detection provided by this type of tracer since resection of the SLN is facilitated thanks to the visualization of elevated contrast enhancement provided by ICG after having performed the dissection of the fatty lymph tissue guided by the acoustic signal of the gamma detector probe by the radioactive signal.

There was an absence of preoperative drainage in 16 patients. In six women there was a higher uptake in bone marrow or leakage to the peritoneum. The latter can be explained by the learning curve of the different ultrasonographers, since this is an operator-dependent technique, and there is an association between ultrasonographer experience and the rate of preoperative SLN detection [6]. Among the 10 remaining patients showing an absence of drainage, lymph node infiltration was detected in the lymphadenectomy in three, and two of these three patients had pelvic nodes metastasis, which was interpreted as a metastatic blockage, while in the remaining patient lymph node metastasis was found in the paraaortic territory. According to the Memorial Sloan Kettering Cancer Center algorithm [21], this should not be considered as a false negative, since in the absence of drainage lymphadenectomy is recommended. However, in this patient the pelvic lymphadenectomy was negative, which would have led to non-completion of the paraaortic lymphadenectomy. This case highlights the importance of the study directed at the paraaortic lymphatic territory.

In two of the 16 patients without preoperative drainage, four SLNs were intraoperatively biopsied by radioactivity and fluorescence. This may be due to the fact on the SPECT/CT, the SLNs with low activity are not correctly visualized by the limit of resolution of the image or are interpreted as non-significant. However, during surgery the greater sensitivity of the gamma probe detects significant activities suggestive of resection. It is therefore always necessary to perform intraoperative detection even though the lymphoscintigraphic images of SPECT/CT do not visualize drainage. Nonetheless, the SPECT/CT in our series was found to significantly increase the detection of SLN with respect to planar lymphoscintigraphy and correlates with surgical detection, as previously reported by other authors $[22,23]$. This allows more directed surgery, which also helps to reduce the number of complications. 
In this study the absence of preoperative drainage was almost $27 \%$. The hybrid tracer has been used in cervical tumors with $100 \%$ preoperative drainage rates and pelvic bilaterality [19]. Therefore, it could be inferred that in contrast to other tumors, other factors could interfere with drainage and the absence of drainage in EC would not be attributable to the hybrid tracer. It is probably due to a multifactorial origin related to EC and this type of myometrial injection, in which the injection dose is inside a very compact and highly vascularized interstitial tissue [6].

The $68.5 \%$ of the SLNs biopsied, were detected by both components. The remaining of the SLNs were identified only by the detection of one of the two components. The lack of detection of fluorescence ( $28.1 \%$ of the SLNs) could be explained by the limit of sensitivity of the NIR optical laparoscopic camera in vivo. Previous articles have referred in vivo detection rates lower than ex vivo rates due to attenuation of the fluorescence signal by the adjacent fatty tissue [17,24]. In our series, ex vivo fluorescence could not be determined with a NIR optical camera for open field detection, which is more sensitive to room light and could have reduced this discrepancy in these cases [25]. The lack of radioactive detection (3.4\%) might have been due to decay of the $\left[{ }^{99 \mathrm{~m}} \mathrm{Tc}\right]$ obtaining a number of non-significant counts in vivo. In SLNs with low presurgical activity and with procedures in which there is a long interval between injection and surgery, it is possible that this activity cannot be detected with the gamma probe whether due to low activity or a scarce SLN/background ratio. To the contrary, fluorescence does not decay provided that it is not exposed to excess excitation light. In four of the five SLNS detected only with the fluorescence signal, the light emission was of great value since in one patient the drainage became bilateral, while in another patient without drainage at least one SLN was biopsied, and in another patient paraaortic SLNs were obtained. This demonstrates that the development of intraoperative fluorescence detector devices and the development and clinical implementation of new tracers are essential to improve and advance surgical efficacy. In addition, it cannot be ruled out that with TUMIR injection there may be a certain dissociation of the hybrid tracer which has not, to date, been described in other tumors, and which may be related to the contact with blood, physiological saline and dilution in general. As a possible explanation we hypothesize that it could be due to the injection being made in the myometrium, which is a highly vascularized structure probably with lesser volume of interstitial tissue, requires a greater volume of injected tracer.

Many studies have used ICG exclusively for visual identification of the SLN in EC, with detection rates greater than $96 \%[26,27]$. One of the drawbacks of ICG is that the fluorescence signal is easily attenuated by the surrounding fatty tissue, unlike the radiotracer signal. That is why the radiotracer linked to ICG allows a quick and effective identification of the SLN during the search through the fatty tissue. Another of the limitations of the ICG is that it has a rapid diffusion through the lymphatic system and stains all the lymphatics structures of a territory, making it difficult to discern the true SLN since it is not always the first visible lymph nodes, which results in 7-8\% of empty packets [28]. In addition, it impedes obtaining a preoperative lymphatic mapping. This can be corrected if it is injected together with a colloid such as $\left[{ }^{99 \mathrm{~m}} \mathrm{Tc}\right] \mathrm{Tc}$-albumin nanocolloid, which allows it to deposit and remain retained in a greater quantity in the first lymph node that receive tumor drainage, thereby identifying the true SLN. 
For these reasons we consider that the radioactive component of the hybrid tracer ( $\left[{ }^{99 \mathrm{~m}} \mathrm{Tc}\right] \mathrm{Tc}$-albumin nanocolloid) cannot be obviated since it provides the route map and together with the ICG signal serves an a multimodal surgical guide towards the true SLN, using a single component and even showing the less usual drainage territories [29]. This hybrid tracer is, thus, of special interest in cases of uterine tumors due to the presence of a complex network of pelvic lymphatic vessels in which knowledge of drainage prior to surgery helps in surgical planning.

Furthermore, in our series, the radioactive signal was the only detection tool that allowed the identification of SLN in more than $17 \%$ of patients, so we recommend not ruling out this component in ES surgeries.

In the accuracy analysis of this study the sensitivity and the NPV obtained for the detection of node metastasis was $100 \%$ in women with intermediate- and high-risk EC. These values are greater than those reported in previous studies in high-risk EC after cervical injection, with sensitivities ranging from 57 to $96.3 \%$ and a NPV of $83.3 \%$ to $99 \%$ [30-32] and are especially greater than hysteroscopic (58 to $100 \%$ and 89 to $100 \%$, respectively) and myometrial injection (75 to $100 \%$ and 96 to $100 \%$, respectively)[33]. The rate of SLN infiltration observed in our series was $14.3 \%$. The current recommendations indicate that performing systematic pelvic and paraaortic lymphadenectomy implies overtreatment in up to $80 \%$ of patients who do not have lymphatic involvement. In addition, one recent study suggested that adding lymphadenectomy to the study of the SLN does not reduce the risk of relapse [34]. On the other hand, patients with paraaortic metastasis have worse oncological results than those with exclusive pelvic lymphatic involvement and, therefore, it is essential to evaluate the status of paraaortic SLN [35].

The present study has some limitations. Among these, this study was performed in a single center and therefore may lack external validity. Among other limitations, it was of note that it was not possible to perform ex vivo detection of fluorescence of the biopsied SLNs with a NIR optical camera optimized for the detection of fluorescence in room light, and therefore, we do not know if the in vivo non fluorescent radioactive SLNS would have been fluorescent by ex vivo stimulation with this another camera. The reduced number of patients is another limitation. However, one of the main strengths of this study was that it was a homogeneous group of patients with intermediate- and high-risk EC and all the surgeries were performed by the same surgical team. In addition, in the present study, all the patients underwent complete pelvic and paraaortic lymphadenectomy up to the renal vessels, in contrast to other studies evaluating the validity of SLNB in high-risk EC [36,37].

Although the percentage of patients with drainage who could undergo intraoperative detection of the SLN was low (around 70\%), the low rate of false negatives is promising. However, multicenter studies are needed to confirm these results and validate that this procedure reduces morbidity, surgical time and costs. The hybrid tracer injected via TUMIR can obtain reliable information on the status of paraaortic lymphs nodes and adapt the adjuvant treatment without the surgical morbidity of systematic paraaortic lymphadenectomy. Based on the recommendations of the clinical guidelines and the results of this study, the strategy to increase the number of patients undergoing intraoperative SLNB would be to inject the hybrid tracer into the cervix in patients without drainage. This would at least provide a map of pelvic 
lymphatic drainage and thereby avoid lymphadenectomy. If this option were not possible, double puncture (myometrial and cervical) of the hybrid tracer could be considered in all patients.

To our knowledge this is the first study on the use of the hybrid tracer for the detection of SLNs in EC, which, furthermore, is injected via TUMIR. Our results of the use of this hybrid tracer confirm the feasibility of this procedure and are consistent with those described in prostate and cervical cancer $[17,19]$. The hybrid tracer adds the benefits of presurgical planning with a dual intraoperative guidance, allowing a potential increase in SLN detection rate (up to $20 \%$ of patients in our series).

\section{Conclusions}

SLNB with a hybrid tracer injected by the TUMIR approach is feasible in intermediate- and high-risk EC. The hybrid tracer allows, in a single injection, the well-established benefits of the standard radioguided procedure and the visualization of SLNs by fluorescence imaging. The use of hybrid tracer by TUMIR injection achieves an elevated paraaortic detection rate and allows a potential increase in SLN detection. Notwithstanding, based on our findings, the radioactive component of the hybrid tracer $\left(\left[{ }^{99 \mathrm{~m}} \mathrm{Tc}\right] \mathrm{Tc}-\right.$ albumin nanocolloid) cannot be obviated.

\section{Declarations}

Funding: None.

Conflicts of interest: None.

Availability of data and material: The datasets generated during and/or analysed during the current study are available from the corresponding author on reasonable request.

Ethics approval: This study was performed in line with the principles of the Declaration of Helsinki. Approval was granted by the Ethics Committee of Hospital Clinic de Barcelona (approval registration number $\mathrm{HCB} / 2019 / 0574$ )

Consent to participate and for publication: Informed consent was obtained from all individual participants included in the study.

Authors' contribution: Conceptualization: N. Sánchez-Izquierdo, S. Vidal-Sicart, P. Paredes. Data curation: N. Sánchez-Izquierdo, M. A. Angeles, A. Perissinotti, F. Campos, M. Munmany, B. Díaz, A. Glickman, A. Saco, J. Ordi, S. Vidal-Sicart, P. Paredes. Formal analysis: F. Migliorelli, M. A. Angeles, N. SánchezIzquierdo, P. Paredes. Investigation: N. Sánchez-Izquierdo, S. Vidal-Sicart, A. Torné, P. Paredes. Methodology: N. Sánchez-Izquierdo, S. Vidal-Sicart, P. Paredes. Project administration: N. SánchezIzquierdo, S. Vidal-Sicart, P. Paredes. Resources: N. Sánchez-Izquierdo, S. Vidal-Sicart, P. Paredes, A. Torné. Supervision: P. Paredes, S. Vidal-Sicart. Visualization: S. Vidal-Sicart, P. Paredes. Writing original draft: N. 
Sánchez-Izquierdo. Writing - review and editing: N. Sánchez-Izquierdo, M. A. Angeles, S. Vidal-Sicart, P. Paredes, M. del Pino. All authors read and approved the final manuscript.

\section{References}

1. Concin N, Matias-Guiu X, Vergote I, et al. ESGO/ESTRO/ESP guidelines for the management of patients with endometrial carcinoma. Int J Gynecol Cancer. 2021;31:12-39.

2. Altman AD, Ferguson SE, Atenafu EG, et al. Canadian high risk endometrial cancer (CHREC) consortium: analyzing the clinical behavior of high risk endometrial cancers. Gynecol Oncol. 2015;139:268-74.

3. Abu-Rustum, NR; Yashar, CM; Bradley, K; et. al. NCCN Guidelines. Uterine Neoplasms. Version 1.2021. 2021.

4. Lecointre L, Lodi M, Faller É, et al. Diagnostic Accuracy and Clinical Impact of Sentinel Lymph Node Sampling in Endometrial Cancer at High Risk of Recurrence: A Meta-Analysis. J Clin Med. 2020;9:3874.

5. Skanjeti A, Dhomps A, Paschetta C, et al. Sentinel Node Mapping in Gynecologic Cancers: A Comprehensive Review. Semin Nucl Med. 2019;49:521-33.

6. Angeles MA, Migliorelli F, León Ramírez LF, et al. Predictive factors of preoperative sentinel lymph node detection in intermediate and high-risk endometrial cancer. Q J Nucl Med Mol Imaging. 2020;64.

7. Van Den Berg NS, Buckle T, Kleinjan Gl, et al. Hybrid tracers for sentinel node biopsy. Q J Nucl Med Mol Imaging. 2014;58:193-206.

8. Guo J, Yang H, Wang S, et al. Comparison of sentinel lymph node biopsy guided by indocyanine green, blue dye, and their combination in breast cancer patients: a prospective cohort study. World J Surg Oncol. 2017;15:196.

9. Ruiz R, Gorostidi M, Jaunarena I, et al. Sentinel node biopsy in endometrial cancer with dual cervical and fundal indocyanine green injection. Int J Gynecol Cancer. 2018;28:139-44.

10. Papadia A, Gasparri ML, Radan AP, et al. Retrospective validation of the laparoscopic ICG SLN mapping in patients with grade 3 endometrial cancer. J Cancer Res Clin Oncol. 2018;144:1385-93.

11. Crane LMA, Themelis G, Pleijhuis RG, et al. Intraoperative multispectral fluorescence imaging for the detection of the sentinel lymph node in cervical cancer: a novel concept. Mol imaging Biol. 2011;13:1043-9.

12. Schaafsma BE, van der Vorst JR, Gaarenstroom KN, et al. Randomized comparison of nearinfrared fluorescence lymphatic tracers for sentinel lymph node mapping of cervical cancer. Gynecol 
Oncol. 2012;127:126-30.

13. Hutteman M, van der Vorst JR, Gaarenstroom KN, et al. Optimization of near-infrared fluorescent sentinel lymph node mapping for vulvar cancer. Am J Obstet Gynecol. 2012;206:89.

14. Brouwer OR, van den Berg NS, Mathéron HM, et al. A hybrid radioactive and fluorescent tracer for sentinel node biopsy in penile carcinoma as a potential replacement for blue dye. Eur Urol. 2014;65:6009.

15. Stoffels I, Dissemond J, Pöppel T, et al. Intraoperative Fluorescence Imaging for Sentinel Lymph Node Detection: Prospective Clinical Trial to Compare the Usefulness of Indocyanine Green vs Technetium Tc 99m for Identification of Sentinel Lymph Nodes. JAMA Surg. 2015;150:617-23.

16. van Leeuwen FWB, Schottelius M, Brouwer OR, et al. Trending: Radioactive and fluorescent bimodal/hybrid tracers as multiplexing solutions for surgical guidance. J Nucl Med. 2020;61:13-9.

17. van der Poel HG, Buckle T, Brouwer OR, et al. Intraoperative Laparoscopic Fluorescence Guidance to the Sentinel Lymph Node in Prostate Cancer Patients: Clinical Proof of Concept of an Integrated Functional Imaging Approach Using a Multimodal Tracer. Eur Urol. 2011;60:826-33.

18. Verbeek FPR, Tummers QRJG, Rietbergen DDD, et al. Sentinel Lymph Node Biopsy in Vulvar Cancer Using Combined Radioactive and Fluorescence Guidance. Int J Gynecol Cancer. 2015;25:108693.

19. Paredes P, Vidal-Sicart S, Campos F, et al. Role of ICG-99mTc-nanocolloid for sentinel lymph node detection in cervical cancer: a pilot study. Eur J Nucl Med Mol Imaging. 2017;44:1853-61.

20. Torné A, Pahisa J, Vidal-Sicart S, et al. Transvaginal ultrasound-guided myometrial injection of radiotracer (TUMIR): a new method for sentinel lymph node detection in endometrial cancer. Gynecol Oncol. 2013;128:88-94.

21. Barlin JN, Khoury-Collado F, Kim CH, et al. The importance of applying a sentinel lymph node mapping algorithm in endometrial cancer staging: beyond removal of blue nodes. Gynecol Oncol. 2012;125:531-5.

22. Perissinotti A, Paredes P, Vidal-Sicart S, et al. Use of SPECT/CT for improved sentinel lymph node localization in endometrial cancer. Gynecol Oncol. 2013;129:42-8.

23. Buda A, Elisei F, Arosio M, et al. Integration of hybrid single-photon emission computed tomography/computed tomography in the preoperative assessment of sentinel node in patients with cervical and endometrial cancer: Our experience and literature review. Int J Gynecol Cancer. 2012;22:8305. 
24. Mathéron HM, van den Berg NS, Brouwer OR, et al. Multimodal surgical guidance towards the sentinel node in vulvar cancer. Gynecol Oncol. 2013;131:720-5.

25. De Grand AM, Frangioni J V. An Operational Near-Infrared Fluorescence Imaging System Prototype for Large Animal Surgery. Technol Cancer Res Treat. 2003;2:553-62.

26. Ruscito I, Gasparri ML, Braicu El, et al. Sentinel Node Mapping in Cervical and Endometrial Cancer: Indocyanine Green Versus Other Conventional Dyes-A Meta-Analysis. Ann Surg Oncol. 2016;23:3749-56.

27. Rossi EC, Kowalski LD, Scalici J, et al. A comparison of sentinel lymph node biopsy to lymphadenectomy for endometrial cancer staging (FIRES trial): a multicentre, prospective, cohort study. Lancet Oncol. 2017;18:384-92.

28. Thomaier $L$, Jager $L$, Stone $R$, et al. Risk of empty lymph node packets in sentinel lymph node mapping for endometrial cancer using indocyanine green. Int J Gynecol Cancer. 2019;29:513-7.

29. Pandit-Taskar N, Gemignani ML, Lyall A, et al. Single photon emission computed tomography SPECT-CT improves sentinel node detection and localization in cervical and uterine malignancy. Gynecol Oncol. 2010;117:59-64.

30. Rajanbabu A, Agarwal R. A prospective evaluation of the sentinel node mapping algorithm in endometrial cancer and correlation of its performance against endometrial cancer risk subtypes. Eur $\mathrm{J}$ Obstet Gynecol Reprod Biol. 2018;224:77-80.

31. Ye L, Li S, Lu W, et al. A Prospective Study of Sentinel Lymph Node Mapping for Endometrial Cancer: Is It Effective in High-Risk Subtypes? Oncologist. 2019;24:1381-7.

32. Persson J, Salehi S, Bollino M, et al. Pelvic Sentinel lymph node detection in High-Risk Endometrial Cancer (SHREC-trial) -the final step towards a paradigm shift in surgical staging. Eur $J$ Cancer. 2019;116:77-85.

33. Holloway RW, Abu-Rustum NR, Backes FJ, et al. Sentinel lymph node mapping and staging in endometrial cancer: A Society of Gynecologic Oncology literature review with consensus recommendations. Gynecol Oncol. 2017;146:405-15.

34. Martinelli F, Ditto A, Bogani G, et al. Sentinel lymph node mapping in endometrial cancer: performance of hysteroscopic injection of tracers. Int J Gynecol Cancer. 2020;30:332-8.

35. Cosgrove CM, Cohn DE, Rhoades J, et al. The prognostic significance of aortic lymph node metastasis in endometrial cancer: Potential implications for selective aortic lymph node assessment. Gynecol Oncol. 2019;153:505-10. 
36. Touhami O, Grégoire J, Renaud MC, et al. Performance of sentinel lymph node (SLN) mapping in high-risk endometrial cancer. Gynecol Oncol. 2017;147:549-53.

37. Ehrisman J, Secord AA, Berchuck A, et al. Performance of sentinel lymph node biopsy in high-risk endometrial cancer. Gynecol Oncol Reports. 2016;17:69-71.

\section{Tables}

Table 1 Postoperative histological characteristics of the 52 patients included in the study

\begin{tabular}{|lc|}
\hline \multicolumn{2}{|l|}{ Histological type and grade } \\
\hline Endometrioid & $38(73.0 \%)$ \\
\hline Grade 1 & $9(23.7 \%)$ \\
Grade 2 & $20(52.6 \%)$ \\
Grade 3 & $9(23.7 \%)$ \\
\hline Serous & $7(13.5 \%)$ \\
Clear cell & $5(9.6 \%)$ \\
\hline Mixed & $2(3.8 \%)$ \\
\hline Histological tumor size \\
\hline$<4$ cm & $27(51.9 \%)$ \\
$\geq$ 4cm & $25(47.2 \%)$ \\
\hline Postoperative FIGO* stage \\
\hline IA & $25(47.2 \%)$ \\
IB & $12(23.0 \%)$ \\
II & $4(7.5 \%)$ \\
IIIA & $0(0.0 \%)$ \\
IIIB & $0(0.0 \%)$ \\
IIIC1 & $5(9.4 \%)$ \\
IIIC2 & $3(5.7 \%)$ \\
IVA & $0(0.0 \%)$ \\
IVB & $3(5.7 \%)$ \\
\hline
\end{tabular}

*FIGO: International Federation of Gynecology and Obstetrics (2009) 
Table 2 Comparison of preoperative detection rate for different techniques and distribution of pelvic and paraaortic SLNs identified in planar lymphoscintigraphic and SPECT/CT images and intraoperative detection

\begin{tabular}{|llll|}
\hline & $\begin{array}{l}\text { Planar Lymphoscintigraphic } \\
\text { DR (n,\%) } \\
25 / 52(48.1 \%)\end{array}$ & $\begin{array}{l}\text { Intraoperative } \\
\text { SPECT/CT DR } \\
\text { detection rate }\end{array}$ \\
$\begin{array}{l}\text { (n,\%) } \\
36 / 52(69.2 \%)\end{array}$ & $\begin{array}{l}35 / 49 *(71.4 \%) \\
\begin{array}{l}\text { Area of SLN } \\
\text { detection }\end{array}\end{array}$ & $23(63.9 \%)$ & $23(65.7 \%)$ \\
$\begin{array}{l}\text { Pelvis (exclusive) } \\
\begin{array}{l}\text { Paraaortic } \\
\text { (exclusive) }\end{array}\end{array}$ & $13(52.0 \%)$ & $0(0.0 \%)$ & $0(0.0 \%)$ \\
$\begin{array}{l}\text { Pelvis and paraaortic } \\
\text { area }\end{array}$ & $12(4.0 \%)$ & $13(36.1 \%)$ & $12(34.3 \%)$ \\
\hline $\begin{array}{l}\text { Side of pelvis } \\
\text { Left }\end{array}$ & $2(8.0 \%)$ & & \\
Right & $7(28.0 \%)$ & $3(8.3 \%)$ & $3(8.6 \%)$ \\
Bilateral & $16(64.0 \%)$ & $13(36.1 \%)$ & $12(34.3 \%)$ \\
\hline
\end{tabular}

DR: preoperative detection rate (drainage on lymphoscintigraphy); SLNs: Sentinel lymph node. SPECT/CT: Single Photon Emission Computed Tomography

* During surgery, three of the 52 patients, showed peritoneal carcinomatosis, so SLN biopsy was ruled out. That results in 49 patients in whom SLN biopsy was attempted

\section{Figures}



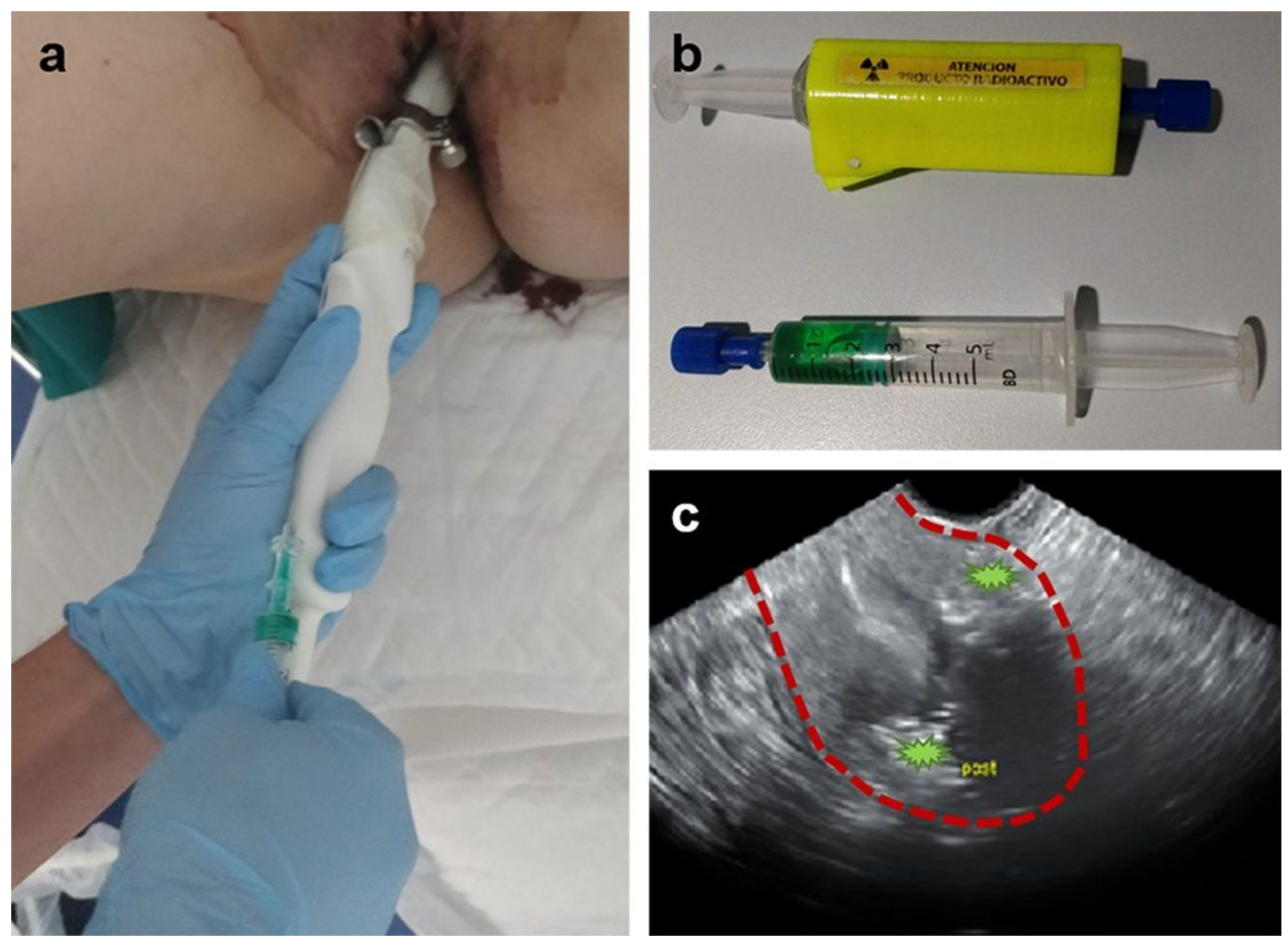

\section{Figure 1}

Transvaginal ultrasound myometrial injection of the hybrid tracer (a). Hybrid tracer (ICG-[99mTc]Tcnanocolloid) prepared for the injection (b). Tracer accumulation (green asterisk) in the anterior and posterior walls of the myometrium. Intermittent red line delimitates the uterus. Red double-headed arrow indicates the endometrium (c) 


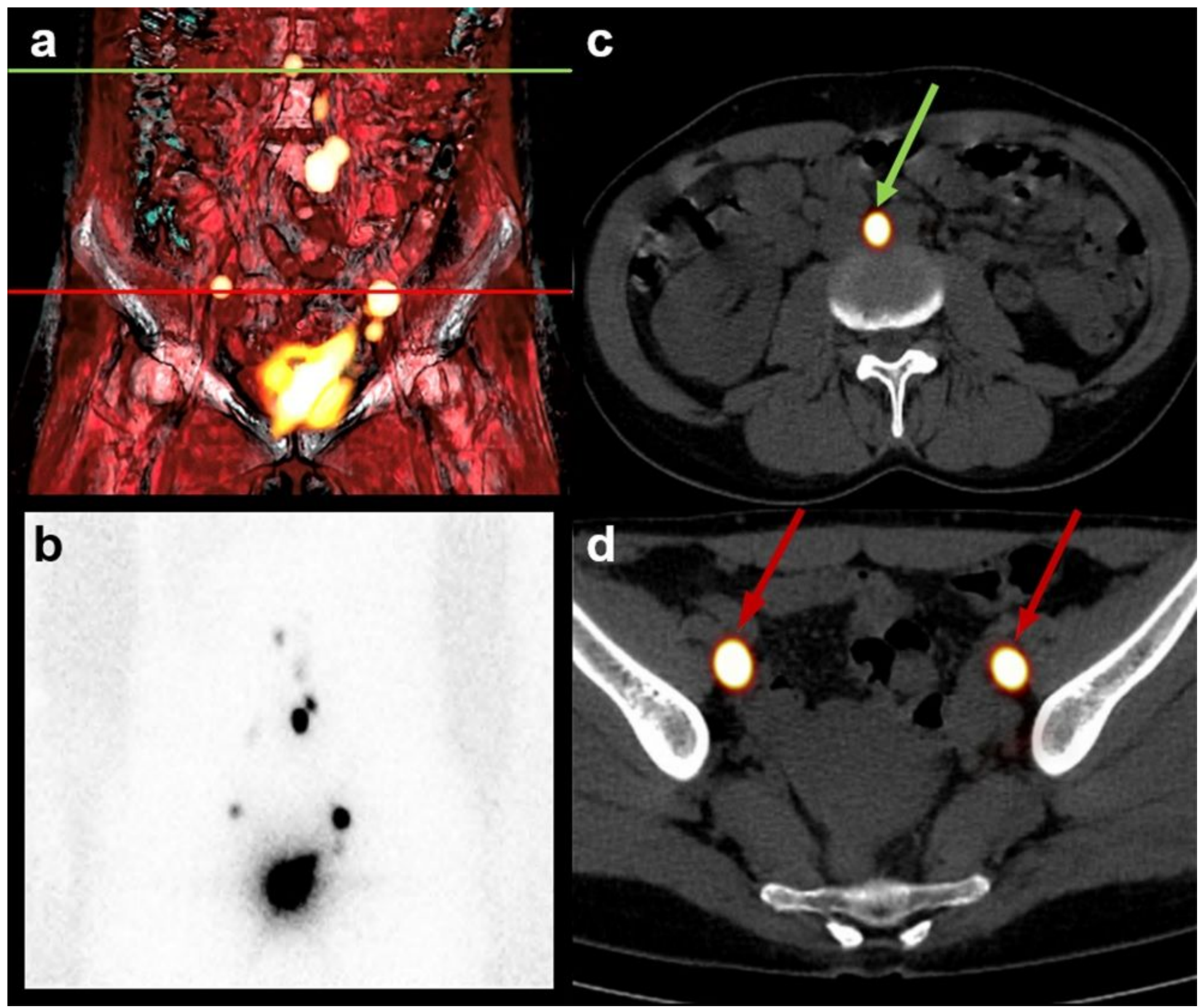

Figure 2

Planar and SPECT/CT lymphoscintigraphy performed $2 \mathrm{~h}$ after TUMIR injection of hybrid tracer (ICG[99mTc]Tc-nanocolloid). Volumetric reconstruction of SPECT/CT (a) and planar lymphoscintigraphy (b) reveal drainage to the bilateral pelvic and paraaortic regions. Axial fused SPECT/CT images showing radioactive SLNs corresponding to a paraaortic node (green arrow) (c) and bilateral external iliac SLNs (red arrows) (d) SPECT/CT: Single Photon Emission Computed Tomography; TUMIR: Transvaginal Ultrasound-guided Myometrial Injection of Radiotracer; SLNs: Sentinel lymph node 

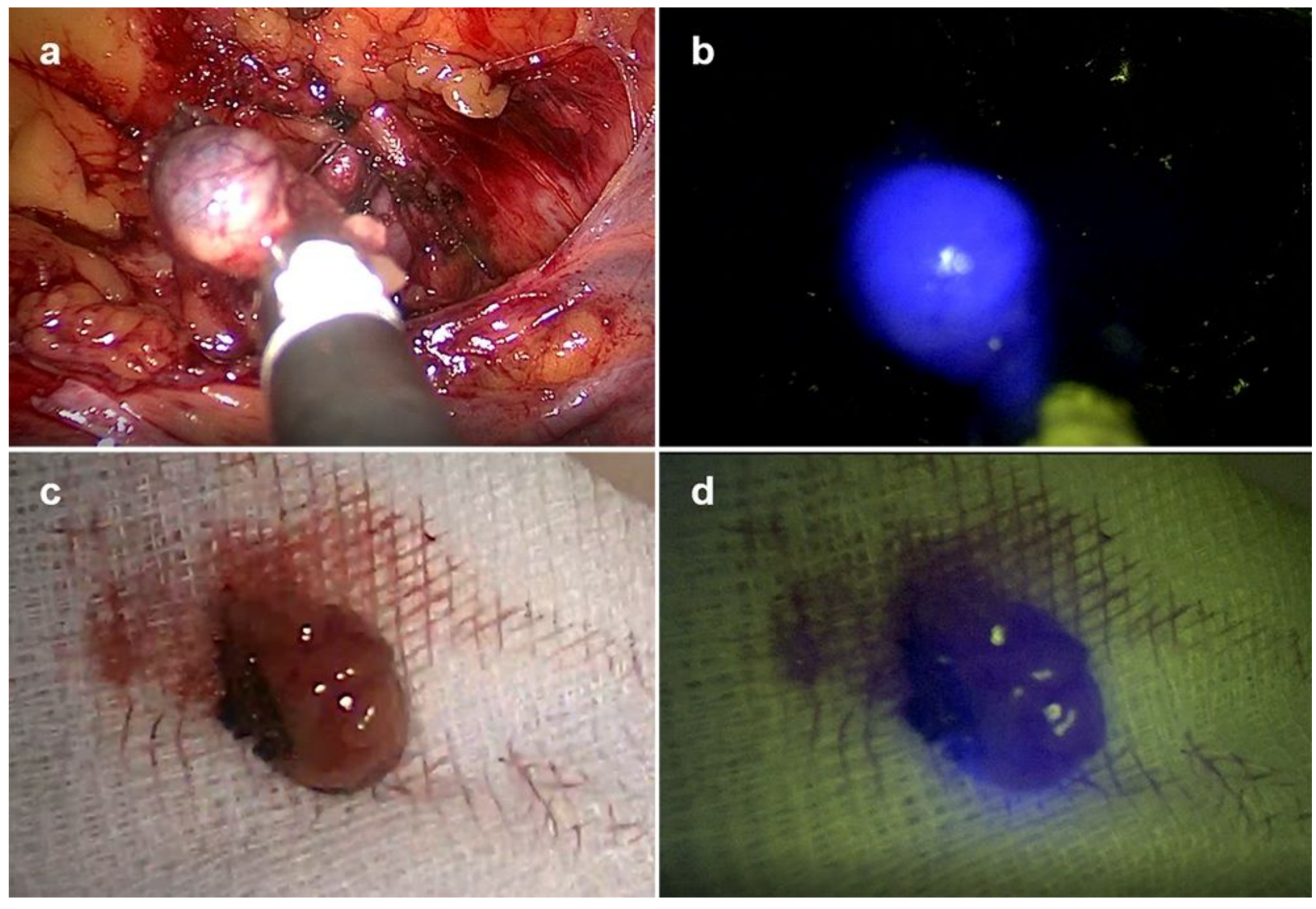

\section{Figure 3}

Intraoperative detection of SLNs by gamma probe and NIR optical camera: Combined laparoscopic sentinel node visualization with white light image (a) and multispectral fluorescence imaging (shown in blue) (b). Ex vivo images of the SLN biopsied with white light (c) and multispectral fluorescence (shown in blue) (d) SLNs: Sentinel lymph node. NIR: Near-infrared 


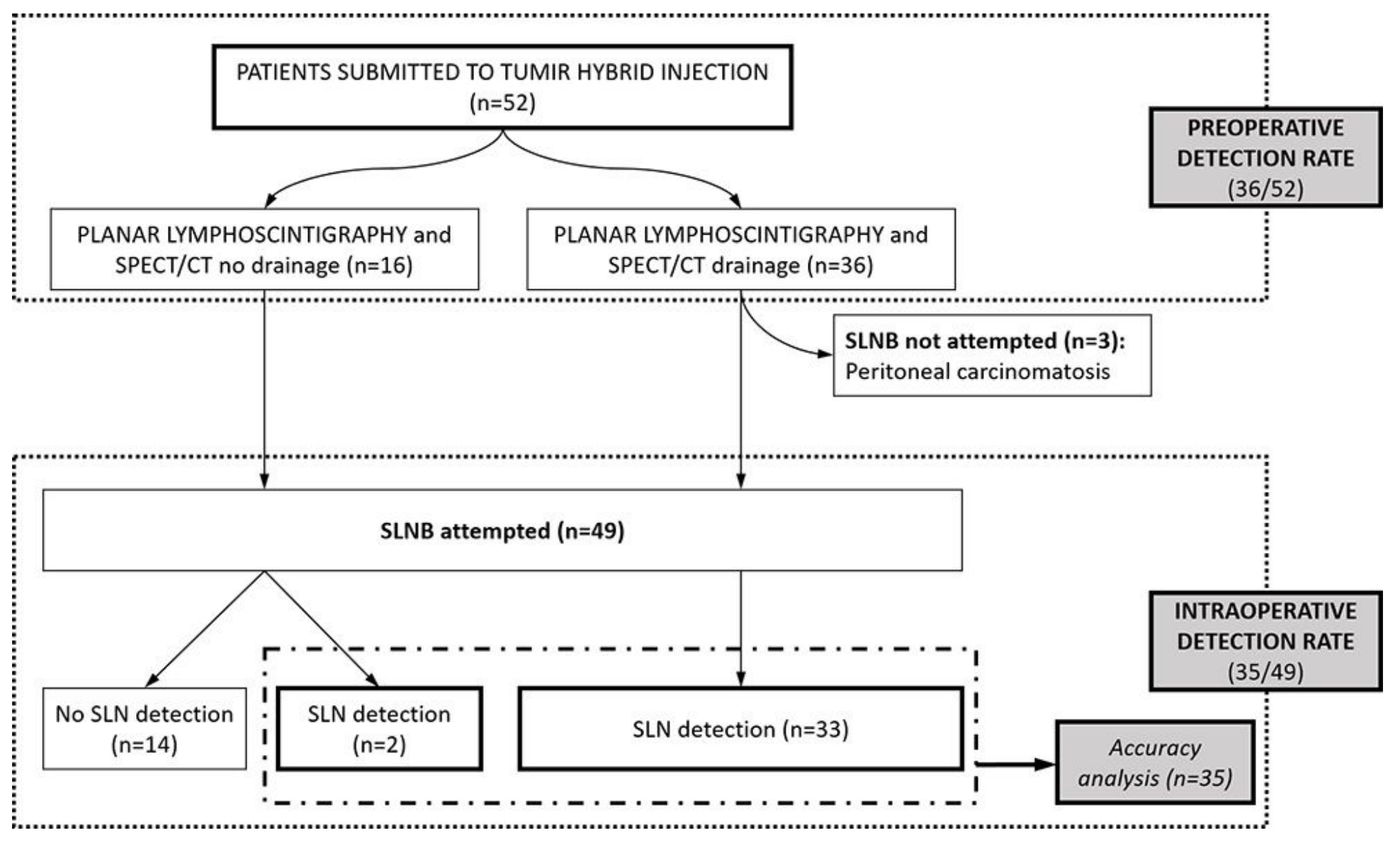

Figure 4

Flow-chart of patients available for analysis and included in the study SPECT/CT: Single Photon Emission Computed Tomography. SLNB: Sentinel lymph node biopsy; SLN: Sentinel Lymph Node 


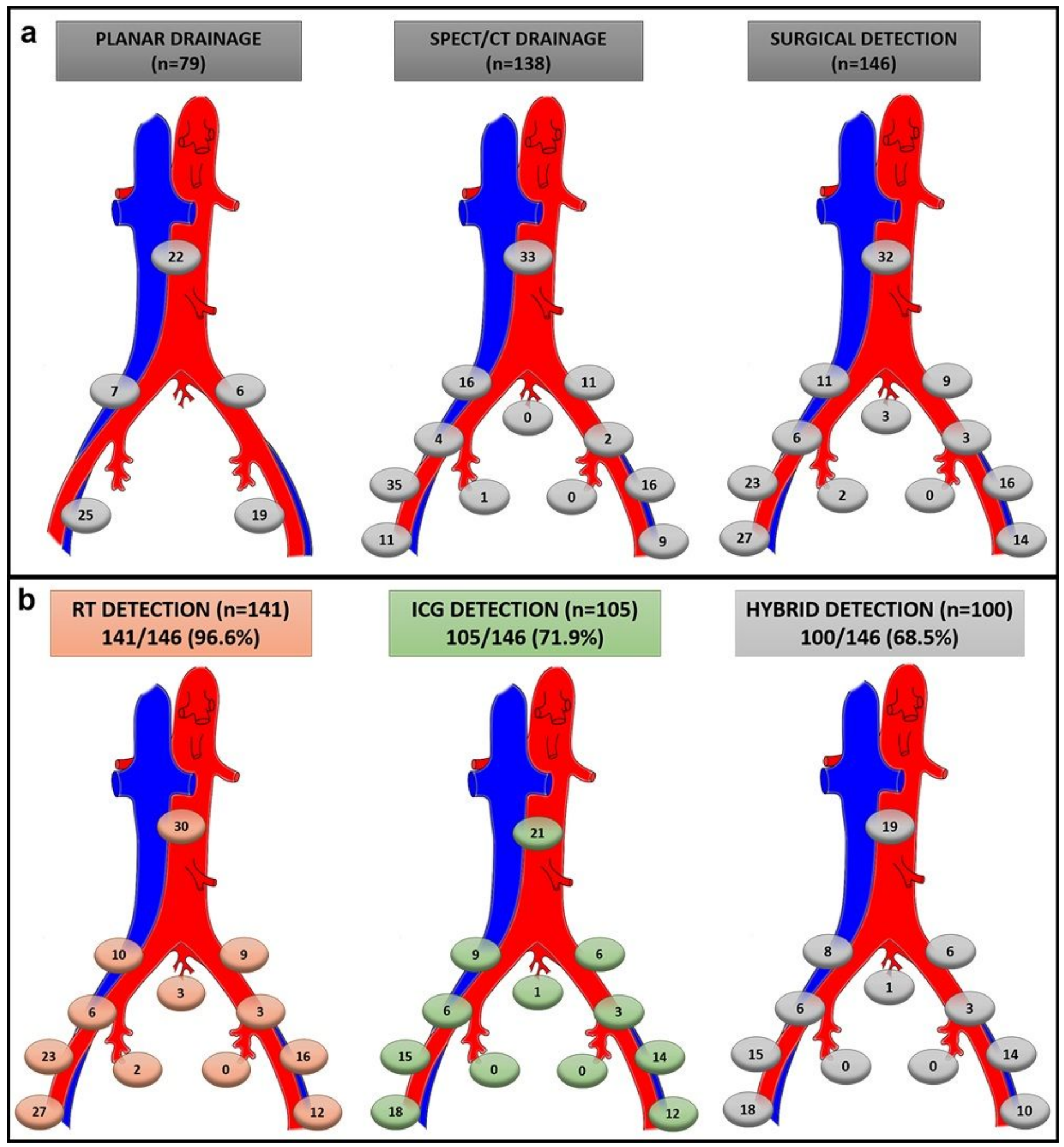

Figure 5

Topographic distribution of SLNs identified in the different anatomical areas on preoperative examination and surgery (a) and depending on the tracer detecting by gamma- and/or NIR-emission (b): radiotracer, indocyanine green and hybrid detection (radiotracer and indocyanine green) SLNs: Sentinel lymph node. NIR: Near-infrared; SPECT/CT: Single Photon Emission Computed Tomography; RT: radiotracer; ICG: indocyanine green 